\title{
Input-to-state Stability of Robust Receding Horizon Control with an Expected Value Cost
}

\author{
Paul J. Goulart ${ }^{\mathrm{a}}$, Eric C. Kerrigan ${ }^{\mathrm{b}}$ \\ ${ }^{a}$ Department of Engineering, University of Cambridge, Trumpington Street, Cambridge CB2 1PZ, UK \\ ${ }^{\mathrm{b}}$ Department of Aeronautics and Department of Electrical and Electronic Engineering, \\ Imperial College London, Exhibition Road, London $S W 72 A Z$, UK
}

\begin{abstract}
This paper is concerned with the stability of a class of receding horizon control laws for constrained linear discrete-time systems subject to bounded state disturbances and compact and convex state and input constraints. The paper considers the class of finite horizon feedback control policies parameterized as affine functions of the system state, calculation of which has recently been shown to be tractable via a convex reparameterization. When minimizing the expected value of a finite horizon quadratic cost, we show that the value function is convex. When solving this optimal control problem at each time step and implementing the result in a receding horizon fashion, we provide sufficient conditions under which the closed-loop system is input-to-state stable (ISS).
\end{abstract}

This paper proposes a class of robust receding horizon control (RHC) laws for constrained linear discrete-time systems subject to bounded state disturbances. We consider a class of affine feedback control policies parameterized as affine functions of the system state, calculation of which has been shown to be tractable via a convex reparameterization [4]. When minimizing the expected value of a finite horizon quadratic function of the states and inputs, we show that the resulting value function is convex and provide sufficient conditions, when used in the design of a RHC law, to establish that the closedloop system is input-to-state stable (ISS). This work is an extension of recent results in [4] to the case where the state and input constraints are non-polyhedral compact and convex sets.

In [4], the authors demonstrate that the non-convex state feedback optimization problem can be reparameterized as an equivalent but convex problem by recasting the optimization problem in terms of affine disturbance or error feedback laws. They further demonstrate that, when implemented in a receding horizon fashion with a particular cost function, the closed-loop system is input-tostate stable (ISS) when the constraints and disturbance sets are polytopic.

In this paper we present a generalization of these results, using the expected value of a quadratic cost. We demonstrate that, for systems with compact and convex state and input constraints and disturbance sets, the resulting value function is convex and lower semicontinuous when optimizing over affine feedback policies, and provide conditions under which input-to-state stability can be established for such systems using convex Lyapunov functions. Since the constraints and disturbance sets we consider are not necessarily polytopic, the proofs differ substantially from those required in the polytopic case. This generalization is of particular interest, for example, in the case where the disturbance set is 2 -norm bounded, and the resultant optimization problem can be solved as a tractable second-order cone program (SOCP), but for which no stability proof exists at present.

Notation: $\mathbb{E}[\cdot]$ is the expectation operator. Given vectors $x$ and $y$ and matrices $A$ and $B,\|x\|:=\sqrt{x^{\prime} x}$, $\operatorname{vec}(x, y):=\left[x^{\prime} y^{\prime}\right]^{\prime},\|A\|_{F}:=\sqrt{\operatorname{trace}\left(A^{\prime} A\right)}$ and $A \otimes B$ is the Kronecker product of $A$ and $B$. Given a set $X$, int $X$ is its interior and $\operatorname{lin} X$ is its linear hull.

\section{Problem Definition}

Consider the following discrete-time linear timeinvariant system:

$$
\begin{aligned}
x^{+} & =A x+B u+G w, \\
z & =C x+D u,
\end{aligned}
$$

where $x \in \mathbb{R}^{n}$ is the system state at the current time instant, $x^{+}$is the state at the next time instant, $u \in \mathbb{R}^{m}$ is the control input, $w \in \mathbb{R}^{n}$ is a disturbance and $z \in \mathbb{R}^{p}$ is a costed/controlled variable. It is assumed that $C^{\prime} D=0$ with $D$ full column $\operatorname{rank},(A, B)$ is stabilizable, $(C, A)$ 
is detectable and that at each sample instant a measurement of the state is available. It is assumed that the current and future values of the disturbance are unknown and may change from one time instant to the next, but are contained in a compact set $W$ containing the origin in its interior, and are independent and identically distributed with zero mean and covariance $\mathbb{E}\left[w w^{\prime}\right]=: C_{w}$. Finally, we assume that the covariance $C_{w}$ is sensibly defined with respect to $G$, i.e. we assume that $\operatorname{null}\left(C_{w}\right) \cap \operatorname{lin}(W)=\{0\}$.

The system is subject to mixed convex constraints on the state and input, so that the system must satisfy $(x, u) \in Z$ where $Z \subset \mathbb{R}^{n} \times \mathbb{R}^{m}$ is a convex and compact set containing the origin in its interior. A design goal is to guarantee that the state and input of the closed-loop system remain in $Z$ for all time and for all allowable disturbance sequences. We further assume that a target/terminal constraint set $X_{f} \subset \mathbb{R}^{n}$ is given, which is convex, compact and contains the origin in its interior.

\subsection{Affine Feedback Control Policies}

For reasons of computational tractability, we elect to work with RHC laws synthesized from the class of finite horizon affine state feedback control policies for the system (1), rather than with arbitrary functions of the state as in [11]. This class of policies parameterizes the future controls inputs $u_{i}$ as $u_{i}=g_{i}+\sum_{j=0}^{i} L_{i, j} x_{j}$, where $x_{0}=x$ denotes the current measured value of the state and $x_{i+1}:=A x_{i}+B u_{i}+G w_{i}$ denotes the prediction of the state after $i$ time instants.

Such an affine parameterization has been shown [4] to be equivalent to the class of control policies parameterized as an affine function of the sequence of past disturbances $[1,7]$, so that

$$
u_{i}=v_{i}+\sum_{j=0}^{i-1} M_{i, j} G w_{j}, \quad \forall i \in\{0, \ldots, N-1\}
$$

for some $M_{i, j} \in \mathbb{R}^{m \times n}$ and $v_{i} \in \mathbb{R}^{m}$. Define the vector $\mathbf{v} \in \mathbb{R}^{m N}$ and the matrix $\mathbf{M} \in \mathbb{R}^{m N \times n N}$ such that

$$
\mathbf{M}:=\left[\begin{array}{cccc}
0 & \cdots & \cdots & 0 \\
M_{1,0} & 0 & \cdots & 0 \\
\vdots & \ddots & \ddots & \vdots \\
M_{N-1,0} & \cdots & M_{N-1, N-2} & 0
\end{array}\right], \mathbf{v}:=\left[\begin{array}{c}
v_{0} \\
v_{1} \\
\vdots \\
v_{N-1}
\end{array}\right] .
$$

Define the set of admissible $(\mathbf{M}, \mathbf{v})$, for which the constraints $Z$ and $X_{f}$ are satisfied, as:

$$
\Pi_{N}(x):=\left\{(\mathbf{M}, \mathbf{v}) \mid \begin{array}{r}
(\mathbf{M}, \mathbf{v}) \text { satisfies }(4), x=x_{0} \\
x_{i+1}=A x_{i}+B u_{i}+G w_{i} \\
u_{i}=v_{i}+\sum_{j=0}^{i-1} M_{i, j} G w_{j} \\
\left(x_{i}, u_{i}\right) \in Z, x_{N} \in X_{f} \\
\forall w_{i} \in W, \forall i \in\{0, \ldots, N-1\}
\end{array}\right\}(5)
$$

and define the set of states for which such an admissible control policy exists as $X_{N}:=\left\{x \in \mathbb{R}^{n} \mid \Pi_{N}(x) \neq \emptyset\right\}$. The parameterization (3) is of particular interest because the set $\Pi_{N}(x)$ is convex [4], whereas the set of feasible state feedback parameters is non-convex, in general. Furthermore, policies in this class can, in certain cases, provide a strictly larger region of attraction than policies based on perturbations to fixed linear feedback laws as in $[2,6,8]$, regardless of horizon length.

\subsection{An Expected Value Cost Function}

We employ a cost function that is quadratic in the state and control sequence, and seek a control policy that will minimize its expected value over the planning horizon. We define

$$
V_{N}(x, \mathbf{M}, \mathbf{v}):=\mathbb{E}\left[\left\|x_{N}\right\|_{P}^{2}+\sum_{i=0}^{N-1}\left\|z_{i}\right\|^{2}\right]
$$

where $P$ is positive definition and $z_{i}=C x_{i}+D u_{i}$ for all $i$. We define the value function $V_{N}^{*}: X_{N} \rightarrow \mathbb{R}_{+}$to be

$$
V_{N}^{*}(x):=\inf _{(\mathbf{M}, \mathbf{v}) \in \Pi_{N}(x)} V_{N}(x, \mathbf{M}, \mathbf{v}) .
$$

We define an optimal policy $\left(\mathbf{M}^{*}(x), \mathbf{v}^{*}(x)\right)$ to be a minimizer of (7) and assume for the moment that such a minimizer exists. We first demonstrate that $V(\cdot, \cdot, \cdot)$ is convex, so that (7) represents a convex optimization problem.

Proposition 1 (Convex Cost Function) The function $(x, \mathbf{M}, \mathbf{v}) \mapsto V_{N}(x, \mathbf{M}, \mathbf{v})$ is convex and quadratic in the state $x$ and parameter $\mathbf{M}$, and strictly convex and quadratic in the parameter $\mathbf{v}$.

Proof. First rewrite (6) in the more compact form

$V_{N}(x, \mathbf{M}, \mathbf{v})=\mathbb{E}\left[\left\|H_{x} x+H_{u} \mathbf{v}+\left(H_{u} \mathbf{M} \mathcal{G}+H_{w}\right) \mathbf{w}\right\|^{2}\right]$

where $\mathbf{w}:=\operatorname{vec}\left(w_{0}, \ldots, w_{N-1}\right)$ and $H_{x}, H_{u}$ and $H_{w}$ are easily constructed from the problem data. Note that since $D$ is assumed to have full column rank, $H_{u}$ also has full column rank. Since $\mathbb{E}[\mathbf{w}]=0$ and $\mathbf{w}$ is independent of both $\mathbf{v}$ and $\mathbf{M}$,

$V_{N}(x, \mathbf{M}, \mathbf{v})=\left\|H_{x} x+H_{u} \mathbf{v}\right\|^{2}+\left\|\left(H_{u} \mathbf{M} \mathcal{G}+H_{w}\right) \mathbf{C}_{w}^{\frac{1}{2}}\right\|_{F}^{2}$,

where $\mathbf{C}_{w}:=I_{N} \otimes C_{w}$, and we have exploited the identity $\mathbb{E}\left[\mathbf{w}^{\prime} X \mathbf{w}\right]=\left\|\left(X \mathbf{C}_{w}^{\frac{1}{2}}\right)\right\|_{F}^{2}$ for any $X$. The result follows immediately, since both $\|\cdot\|$ and $\|\cdot\|_{F}$ are strictly convex functions and $H_{u}$ has full column rank.

If the constraint sets $Z$ and $X_{f}$ are polytopic, then (7) can be expressed as an SOCP when $W$ is ellipsoidal, and as a quadratic program (QP) when $W$ is polytopic, both in a polynomial number of variables and constraints [3] 


\section{Stability of RHC Law}

We define a receding horizon control law $\mu_{N}: X_{N} \rightarrow \mathbb{R}^{m}$ using the first part of the optimal affine feedback control policy $\left(\mathbf{M}^{*}(x), \mathbf{v}^{*}(x)\right.$, i.e. $\mu_{N}(x):=v_{0}^{*}(x)$, where $\mathbf{v}^{*}(x)=: \operatorname{vec}\left(v_{0}^{*}(x), \ldots, v_{N-1}^{*}(x)\right)$ - note that this control law is time-invariant and is a nonlinear function of the current state in general.

We wish to find conditions under which the closed-loop system

$$
x^{+}=A x+B \mu_{N}(x)+G w
$$

is input-to-state stable (ISS) [5]. To do this, we require some results related to the convexity of the value function $V_{N}^{*}(\cdot)$ in $(7)$, and to input-to-state stability for systems with convex ISS-Lyapunov functions. We first demonstrate that the value function $V_{N}^{*}(\cdot)$ in $(7)$ is convex and continuous on the interior of its domain; this property will prove useful in our subsequent proof of stability for the closed-loop system (8).

Proposition 2 (Continuity and convexity of $V_{N}^{*}(\cdot)$ and $\left.\mu_{N}(\cdot)\right)$ If $X_{N}$ has a non-empty interior, then $V_{N}^{*}(\cdot)$ is convex on $X_{N}$, continuous on int $X_{N}$ and lower semicontinuous everywhere on $X_{N}$. The $R H C$ law $\mu_{N}(\cdot)$ is unique on $X_{N}$ and continuous on $\operatorname{int} X_{N}$.

Proof. Define $\mathcal{C}_{N}:=\left\{(x, \mathbf{M}, \mathbf{v}) \mid(\mathbf{M}, \mathbf{v}) \in \Pi_{N}(x)\right\}$, which is a closed and convex set, and which can be written as the Minkowski sum of a compact set and a subspace when $Z$ is compact [3]. Further define the set

$\mathcal{V}_{N}:=\left\{(x, \mathbf{Y}, \mathbf{v}) \mid \exists \mathbf{M}, \mathbf{Y}=\mathbf{M} \mathcal{G} \mathbf{C}_{w}^{\frac{1}{2}},(x, \mathbf{M}, \mathbf{v}) \in \mathcal{C}_{N}\right\}$

Since the mapping $\mathcal{C}_{N} \mapsto \mathcal{V}_{N}$ is linear, the set $\mathcal{V}_{N}$ is also closed and convex [9, Thm 9.1]. Define the extended real-valued function

$$
h(x, \mathbf{Y}, \mathbf{v}):= \begin{cases}\tilde{V}_{N}(x, \mathbf{Y}, \mathbf{v}) & (x, \mathbf{Y}, \mathbf{v}) \in \mathcal{V}_{N} \\ \infty & \text { otherwise }\end{cases}
$$

where

$$
\tilde{V}_{N}(x, \mathbf{Y}, \mathbf{v}):=\left\|H_{x} x+H_{u} \mathbf{v}\right\|^{2}+\left\|\left(H_{u} \mathbf{Y}+H_{w} \mathbf{C}_{w}^{\frac{1}{2}}\right)\right\|_{F}^{2},
$$

so that (9) is convex, lower semicontinuous and proper. Since $H_{u}$ is full column rank, $h(x, \mathbf{Y}, \mathbf{v})$ is strictly convex in $(\mathbf{Y}, \mathbf{v})$ and level bounded in $x$ locally uniformly in $(\mathbf{Y}, \mathbf{v})$ - see [10, Defn 1.16]. Rewriting the value function $V_{N}^{*}(\cdot)$ as

$$
V_{N}^{*}(x)=\inf _{(\mathbf{Y}, \mathbf{v})} h(x, \mathbf{Y}, \mathbf{v})
$$

with associated minimizer $\left(\mathbf{Y}^{*}(x), \mathbf{v}^{*}(x)\right)$, it follows that $V_{N}^{*}(\cdot)$ is convex and lower semicontinuous [10, Prop. 1.17] on $X_{N}$ and strictly continuous on $\operatorname{int} X_{N}$ [10, Thm 2.35]. The function $\mathbf{v}^{*}(\cdot)$ is single-valued on $X_{N}$ and continuous on $\operatorname{int} X_{N}$ [10, Thm 3.31 \& Cor. 7.43]. The uniqueness and continuity of $\mu_{N}(\cdot)$ then follow directly.
Corollary 3 The function $V_{N}(x, \cdot, \cdot)$ attains its minimum on the set $\Pi_{N}(x)$.

We next develop a result on the input-to-state stability of systems with convex value functions. We can then exploit the convexity of the value function $V_{N}^{*}(\cdot)$ to provide conditions in which the closed-loop system (8) is inputto-state stable (ISS) when implemented in a receding horizon fashion.

Consider a nonlinear, time-invariant, discrete-time system of the form

$$
x^{+}=f(x, w),
$$

where $x \in \mathbb{R}^{n}$ is the state and $w \in \mathbb{R}^{l}$ is a disturbance that takes on values in a compact set $W \subset \mathbb{R}^{l}$ containing the origin. It is assumed that the state is measured at each time instant, that $f: \mathbb{R}^{n} \times \mathbb{R}^{l} \rightarrow \mathbb{R}^{n}$ is continuous at the origin and that $f(0,0)=0$. Given an initial state $x$ and a disturbance sequence $w(\cdot)$, where $w(k) \in W$ for all $k \in \mathbb{N}$, let the solution to (10) at time $k$ be denoted by $\phi(k, x, w(\cdot))$. For systems of this type, a useful definition of stability is input-to-state stability:

Definition 4 (ISS) The system (10) is input-to-state stable (ISS) in $\mathcal{X} \subseteq \mathbb{R}^{n}$ if there exist a $\mathcal{K} \mathcal{L}$-function $\beta(\cdot)$ and $a \mathcal{K}$-function $\gamma(\cdot)$ such that for all initial states $x \in \mathcal{X}$ and disturbance sequences $w(\cdot)$, where $w(k) \in W$ for all $k \in \mathbb{N}$, the solution of the system satisfies $\phi(k, x, w(\cdot)) \in$ $\mathcal{X}$ and for all $k \in \mathbb{N}$,

$$
\begin{aligned}
& \|\phi(k, x, w(\cdot))\| \leq \beta(\|x\|, k)+ \\
& \gamma(\sup \{\|w(\tau)\| \mid \tau=\{0, \ldots, k-1\}\})
\end{aligned}
$$

Proposition 5 (Convex Lyapunov function for undisturbed system) Let $\mathcal{X} \subseteq \mathbb{R}^{n}$ be a compact robust positively invariant set for (10) containing the origin in its interior. Furthermore, let there exist $\mathcal{K}_{\infty}$-functions $\alpha_{1}(\cdot), \alpha_{2}(\cdot)$ and $\alpha_{3}(\cdot)$ and a function $V: \mathcal{X} \rightarrow \mathbb{R}_{\geq 0}$ that is convex on $\mathcal{X}$ such that for all $x \in \mathcal{X}$,

$$
\begin{aligned}
\alpha_{1}(\|x\|) & \leq V(x) \leq \alpha_{2}(\|x\|) \\
V(f(x, 0)) & -V(x) \leq-\alpha_{3}(\|x\|)
\end{aligned}
$$

The function $V(\cdot)$ is an ISS-Lyapunov function in the sense of [5, Lem. 3.5], and the system (10) is ISS in $\mathcal{X}$ if $f(\cdot)$ can be written as $f(x, w):=g(x)+w$, where $g(\cdot)$ is continuous at the origin with $g(0)=0$ and $W$ is compact and convex, containing the origin in its relative interior.

Proof. It is sufficient to show that there exists a constant $\gamma$ such that $V(f(x, w))-V(f(x, 0)) \leq \gamma\|w\|$ for all $x \in \mathcal{X}$ and all $w \in W$. It then follows that $V(f(x, w))-$ $V(x)=V(f(x, 0))-V(x)+V(f(x, w))-V(f(x, 0)) \leq$ $-\alpha_{3}(\|x\|)+\gamma\|w\|$, and the conditions of [5, Lem. 3.5] are satisfied.

When the disturbance set $W$ is compact and contains the origin in its (relative) interior, there exists a constant $\rho>0$ such that $\rho:=\max \left\{\epsilon \mid\left(\mathcal{B}_{\epsilon} \cap \operatorname{lin} W\right) \subseteq W\right\}$, 
where $\mathcal{B}_{\epsilon}:=\{x \mid\|x\| \leq \epsilon\}$, so that $\rho$ is the size of the smallest vector on the (relative) boundary of $W$. Since the set $\mathcal{X}$ is compact, (12a) implies that $V$ is upper bounded by a constant $b$ and lower bounded by 0 . Since the set $\mathcal{X}$ is robust positively invariant, it follows that $g(x) \in \tilde{\mathcal{X}}:=\left\{x \in \mathbb{R}^{n} \mid x+w \in \mathcal{X}, \forall w \in W\right\}$. Finding a suitable $\gamma$ is equivalent to finding one that satisfies $V(\tilde{x}+w)-V(\tilde{x}) \leq \gamma\|w\|$ for all $\tilde{x} \in \mathcal{X}$ and all $w \in W$. Since $W$ is convex and compact, for any given $w \in W$ there exists a $\tilde{w}$ on the (relative) boundary of $W$ such that $w=\tau \tilde{w}$ with $0 \leq \tau \leq 1$. Note also that $\tau=\|w\| /\|\tilde{w}\| \leq\|w\| / \rho$. Since $\mathcal{X}$ is robust positively invariant, $\tilde{x}+\tilde{w} \in \mathcal{X} \forall \tilde{x} \in \tilde{\mathcal{X}}$. Since $V$ is convex, it follows that $V(\tilde{x}+w) \leq(1-\tau) V(\tilde{x})+\tau V(\tilde{x}+\tilde{w})$, or $V(\tilde{x}+w)-V(\tilde{x}) \leq \tau(V(\tilde{x}+\tilde{w})-V(\tilde{x})) \leq(b / \rho)\|w\|$. The proof is completed by selecting $\gamma:=b / \rho$.

We can now provide conditions under which the closedloop system (8) is guaranteed to be ISS. We first make the following standard assumption:

A1 (Terminal Cost and Constraint) The terminal constraint set $X_{f}$ is both constraint admissible and robust positively invariant under the control $u=K x$. We further assume that the feedback matrix $K$ and terminal cost function $P$ are derived from the solution to the discrete algebraic Riccati equation:

$$
\begin{aligned}
& P:=C^{\prime} C+A^{\prime} P A-K^{\prime}\left(D^{\prime} D+B^{\prime} P B\right) K \\
& K:=-\left(D^{\prime} D+B^{\prime} P B\right)^{-1} B^{\prime} P A .
\end{aligned}
$$

Using the above assumption, the following result regarding the behavior of the undisturbed closed-loop system can be established. The proof uses arguments similar to those in [4] and is omitted for brevity.

Lemma 6 ([3, Lem. 4.18] Properties of $\mu_{N}(\cdot)$ and $\left.V_{N}^{*}(0)\right)$ If $\boldsymbol{A} \mathbf{1}$ holds, then the following conditions hold:

a) The $\mathrm{RHC}$ law $\mu_{N}(\cdot)$ satisfies $\mu_{N}(0)=0$.

b) There exists a positive constant $k_{1}$ such that $V_{N}^{*}\left(A x+B \mu_{N}(x)\right)-V_{N}^{*}(x) \leq-k_{1}\|x\|_{2}^{2}$.

c) There exist positive constants $k_{2}$ and $k_{3}$ such that $k_{2}\|x\|_{2}^{2} \leq\left(V_{N}^{*}(x)-V_{N}^{*}(0)\right) \leq k_{3}\|x\|_{2}^{2}$.

d) The undisturbed closed-loop system $x^{+}=A x+B \mu_{N}(x)$ is exponentially stable in $X_{N}$.

Theorem 7 (ISS for RHC) If $\boldsymbol{A} 1$ holds, then the closed-loop system (8) is ISS in $X_{N}$. Furthermore, the input and state constraints are satisfied for all time and all allowable disturbance sequences if and only if the initial state is in $X_{N}$.

Proof. Define $V(\cdot)=V_{N}^{*}(\cdot)-V_{N}^{*}(0)$, and let $\Omega:=G W$ and $f(x, w):=A x+B \mu_{N}(x)+G w$. If $\mathbf{A} \mathbf{1}$ holds, then the set $X_{N}$ is robust positively invariant for system (8), with $0 \in \operatorname{int} X_{N}$ [4]. The set $X_{N}$ is also easily shown to be compact when $\mathrm{Z}$ is compact [3]. From Proposition 2 the function $V_{N}^{*}(\cdot)$ is convex and continuous on $\operatorname{int}\left(X_{N}\right)$. The remainder of the proof follows by direct application of the results in Lemma 6 and Proposition 5.

\section{Conclusions}

Using a finite horizon affine feedback policy parameterization and exploiting the results in [4], we have shown that receding horizon control laws can be constructed that guarantee input-to-state stability for systems with general compact and convex state and input constraints, given appropriate terminal conditions. The method is based on minimization of the expected value of a finite horizon quadratic cost at each time instant. Central to this result is a proof of the existence of minimizers and convexity of the value function in the underlying finite horizon optimal control problem, by using results from variational analysis. We also provide conditions under which input-to-state stability may be established using convex Lyapunov functions. This result represents an important generalization of the results in [4], as it establishes stability for a broad class of RHC problems using this framework with non-polytopic convex disturbance sets (e.g. ellipsoidal or 2-norm bounded disturbances), or for problems with non-polyhedral convex constraints on the states and inputs.

\section{References}

[1] A. Ben-Tal, A. Goryashko, E. Guslitzer, and A Nemirovski. Adjustable robust solutions of uncertain linear programs. Mathematical Programming, 99(2):351-376, 2004.

[2] L. Chisci, J. A. Rossiter, and G. Zappa. Systems with persistent state disturbances: predictive control with restricted constraints. Automatica, 37(7):1019-1028, 2001.

[3] P. J. Goulart. Affine Feedback Policies for Robust Control with Constraints. PhD thesis, University of Cambridge, 2006. Available at http://www-control.eng.cam.ac.uk.

[4] P. J. Goulart, E. C. Kerrigan, and J. M. Maciejowski. Optimization over state feedback policies for robust control with constraints. Automatica, 42(4):523-533, 2006.

[5] Z. Jiang and Y. Wang. Input-to-state stability for discretetime nonlinear systems. Automatica, 37(6):857-869, 2001.

[6] Y. I. Lee and B. Kouvaritakis. Constrained receding horizon predictive control for systems with disturbances. International Journal of Control, 72(11):1027-1032, 1999.

[7] J. Löfberg. Minimax Approaches to Robust Model Predictive Control. PhD thesis, Linköping University, 2003.

[8] D. Q. Mayne, M. M. Seron, and S. V. Raković. Robust model predictive control of constrained linear systems with bounded disturbances. Automatica, 41(2):219-24, 2005.

[9] R. T. Rockafellar. Convex Analysis. Princeton University Press, USA, 1970.

[10] R. T. Rockafellar and R. J-B. Wets. Variational Analysis. Springer-Verlag, 1998.

[11] P. O. M. Scokaert and D. Q. Mayne. Min-max feedback model predictive control for constrained linear systems. IEEE Trans. on Automatic Control, 43(8):1136-1142, 1998. 